\title{
Nonlinear Controller Design for the Underactuated Crane System
}

\author{
Diantong Liu* and Weiping Guo \\ Institute of Computer Science and Technology, Yantai University, Yantai City, \\ Shandong Province, 264005, People's Republic of China \\ diantong.liu@163.com
}

\begin{abstract}
A nonlinear controller is designed for the underactuated crane. In the design process, a nonlinear variable composed with all the system states is defined and then the nonlinear controller is obtained with the Lyapunov stability theory. The system stability is proved via a stability theorem. The simulation results show the system under any initial states is asymptotically stable to the origin and the proposed algorithm is validated.
\end{abstract}

Keywords: Underactuated system, Crane, Nonlinear control, Ttransport control

\section{Introduction}

Crane works as a robot in many places such as workshops and harbors to transport all kinds of massive goods. It is desired for the overhead crane to transport its payloads to the required position as fast and as accurately as possible without collision with other equipments. Moreover, the payload swing angle should be kept as small as possible [1].

In the last two decades, the control problems of crane systems have been a focus. Yoshida [2] proposed a saturating control law by using a guaranteed cost control method for a nominal linearized crane dynamics. Giua [3] considered a linearized parameter-varying model of a planar crane and proposed an observer-based control design via Lyapunov equivalence. Liu [4] investigated an adaptive sliding mode fuzzy control approach for a linearized twodimension overhead crane system. However, these methods based on the linearized crane dynamics may lose the sufficient accuracy and may reduce the performance of the crane control system.

With the development of nonlinear control technology, many nonlinear methods based on the nonlinear model of crane systems have been presented. Yang [5] developed a nonlinear control scheme incorporating parameter adaptive mechanism to ensure the overall closed-loop system stability. Fang and Sun [6,7] presented a nonlinear coupling control approach. Yang [8] presented a robust control approach based on the wave propagation in a crane cable for a gantry crane system with hoisting. Many sliding mode control algorithms are proposed, such as Wang [9], Choi [10], Ngo [11] and Qian [12].

In this paper, the crane system is transformed into a nonlinear system through a collocated partial feedback linearization. A nonlinear variable that is composed of all the system states is defined and the controller is obtained by a selected Lyapunov function. The system stability is studied via a stability theorem. 


\section{The Crane System Model}

The crane is shown schematically in Figure 1 , where, $m_{1}$ is the trolley mass, $m_{2}$ is the load mass, $l$ is the rope length, $\theta$ is the swing angle of the load with respect to the vertical line, $x$ is the trolley position with respect to the origin, $f$ is the force applied to the trolley. Since no rotating torque can drive load swing directly, the crane is a benchmark example of the underactuated mechanical system, which has one control input $f$ and two configuration variables $(x, \theta)$, and its Euler-Lagrange equations of motion [12] can be obtained as

$$
\left\{\begin{array}{l}
\left(m_{1}+m_{2}\right) \ddot{x}+m_{2} l \cos (\theta) \ddot{\theta}-m_{2} l \sin (\theta) \dot{\theta}^{2}=f \\
m_{2} l \cos (\theta) \ddot{x}+m_{2} l^{2} \ddot{\theta}+m_{2} g l \sin (\theta)=0
\end{array}\right.
$$

Through the following collocated partial feedback linearization [13],

$$
f=\left(m_{1}+m_{2} \sin ^{2} \theta\right) u-m_{2} g \sin \theta \cos \theta-m_{2} l \dot{\theta}^{2} \sin \theta
$$

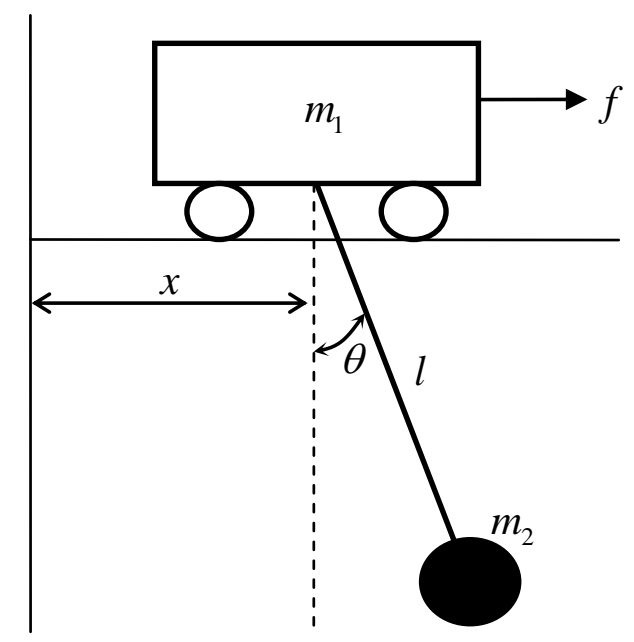

Figure 1. Crane system configuration

The dynamics can be reduced to

$$
\left\{\begin{array}{l}
\ddot{x}=u \\
\ddot{\theta}=-(u \cos \theta+g \sin \theta) / l
\end{array}\right.
$$

\section{The Nonlinear Controller Design}

A nonlinear variable that includes all the system states is defined for the nonlinear underactuated crane system as

$$
s=k_{1} \theta+k_{2} x+k_{3}\left(m_{2} l \dot{x} \cos \theta+m_{2} l^{2} \dot{\theta}\right)
$$

where, $k_{1}, k_{2}$ and $k_{3}$ are some positive constants to assure the system stability. The derivative of the nonlinear variable can be obtained

$$
\dot{s}=k_{1} \dot{\theta}+k_{2} \dot{x}+k_{3}\left(m_{2} l \ddot{x} \cos \theta+m_{2} l^{2} \ddot{\theta}-m_{2} l \dot{x} \dot{\theta} \sin \theta\right)
$$


Form the equation (1), it can be obtained

$$
\dot{s}=k_{1} \dot{\theta}+k_{2} \dot{x}-k_{3}\left(m_{2} g l \sin \theta+m_{2} l \dot{x} \dot{\theta} \sin \theta\right)
$$

In order to obtain a nonlinear controller, the following scalar positive definite Lyapunov function is selected as

$$
V_{1}=\frac{1}{2} s^{2}
$$

Its derivative is

$$
\dot{V}_{1}=s \dot{s}
$$

To make $\dot{V}_{1}$ be a negative definite function, let

$$
\dot{s}=e-b_{1} s
$$

i.e.,

$$
e=\dot{s}+b_{1} s=k_{1} \dot{\theta}+k_{2} \dot{x}-k_{3}\left(m_{2} g l \sin \theta+m_{2} l \dot{x} \dot{\theta} \sin \theta\right)+b_{1} s
$$

Its derivative is

$$
\dot{e}=k_{1} \ddot{\theta}+k_{2} \ddot{x}-k_{3}\left(m_{2} g l \dot{\theta} \cos \theta+m_{2} l \ddot{x} \dot{\theta} \sin \theta+m_{2} l \dot{x} \ddot{\theta} \sin \theta+m_{2} l \dot{x} \dot{\theta}^{2} \cos \theta\right)+b_{1} \dot{s}
$$

From the equation (2), it can be obtained

$$
\begin{aligned}
\dot{e}= & \left(k_{2}-k_{3} m_{2} l \dot{\theta} \sin \theta-k_{1} \cos \theta / l+k_{2} m_{2} \dot{x} \sin \theta \cos \theta\right) u \\
& -g \sin \theta\left(k_{1}-k_{2} m_{2} l \dot{x} \sin \theta\right) / l-k_{2}\left(m_{2} g l \dot{\theta} \cos \theta+m_{2} l \dot{\theta}^{2} \dot{x}\right)+b_{1} \dot{s}
\end{aligned}
$$

The Lyapunov function is reselected to

$$
V=\frac{1}{2} s^{2}+\frac{1}{2} e^{2}
$$

Using (7) and (9), the time derivative $\dot{V}$ is given by

$$
\begin{aligned}
\dot{V}= & s \dot{s}+e \dot{e} \\
= & -b_{1} s^{2}+(s+\dot{e}) e \\
= & -b_{1} s^{2}+\left(s+\left(k_{2}-k_{3} m_{2} l \dot{\theta} \sin \theta-k_{1} \cos \theta / l+k_{2} m_{2} \dot{x} \sin \theta \cos \theta\right) u\right. \\
& \left.-g \sin \theta\left(k_{1}-k_{2} m_{2} l \dot{x} \sin \theta\right) / l-k_{2}\left(m_{2} g l \dot{\theta} \cos \theta+m_{2} l \dot{\theta}^{2} \dot{x} \cos \theta\right)+b_{1} \dot{s}\right) e
\end{aligned}
$$

We note that the variable $u$ enters the right hand side of equation (11). In order to make the $\dot{V}$ be negative definite, we can make the following equation hold

$$
\begin{aligned}
& \left(k_{2}-k_{3} m_{2} l \dot{\theta} \sin \theta-k_{1} \cos \theta / l+k_{2} m_{2} \dot{x} \sin \theta \cos \theta\right) u \\
& \quad-g \sin \theta\left(k_{1}-k_{2} m_{2} l \dot{x} \sin \theta\right) / l-k_{2}\left(m_{2} g l \dot{\theta} \cos \theta+m_{2} l \dot{\theta}^{2} \dot{x} \cos \theta\right)+b_{1} \dot{s}+s=-b_{2} e
\end{aligned}
$$

where $b_{2}$ is a design constant, Such that

$$
\dot{V}=-b_{1} s^{2}-b_{1} e^{2}
$$


Therefore, the control law can be obtained from (12) as

$$
\begin{aligned}
u= & \left(k_{2}-k_{3} m_{2} l \dot{\theta} \sin \theta-k_{1} \cos \theta / l+k_{2} m_{2} \dot{x} \sin \theta \cos \theta\right)^{-1} \\
& \left(g \sin \theta\left(k_{1}-k_{2} m_{2} l \dot{x} \sin \theta\right) / l+k_{2}\left(m_{2} g l \dot{\theta} \cos \theta+m_{2} l \dot{\theta}^{2} \dot{x} \cos \theta\right)-b_{1} \dot{s}-s-b_{2} e\right)
\end{aligned}
$$

Theorem 1: the crane system described by equation (1) is asymptotically stable to its equilibrium point $(x, \dot{x}, \theta, \dot{\theta})=(0,0,0,0)$ under the control input (2) with (14) and some positive constants $k_{1}, k_{2}, k_{3}, b_{1}, b_{2}$.

Proof:

The design process of the nonlinear controller has proved: the time derivative $\dot{V}$ of the chosen positive definite Lyapunov function $V$ is negative definite.

Integrating both sides of (13) yields

$$
\int_{0}^{0} \dot{V} d \tau=\int_{0}^{0}\left(-b_{1} s^{2}-b_{1} e^{2}\right) d \tau
$$

Then

$$
V(t)-V(0)=\int_{0}^{0}\left(-b_{1} s^{2}-b_{1} e^{2}\right) d \tau
$$

We find that

$$
V(t)=\frac{1}{2} s^{2}+\frac{1}{2} e^{2}=V(0)+\int_{0}^{0}\left(-b_{1} s^{2}-b_{1} e^{2}\right) d \tau \leq V(0)<\infty
$$

Therefore it can be obtained that $s \in L_{\infty}$ and $e \in L_{\infty}$, i.e.

$$
\sup |s|=\|s\|_{\infty}<\infty, \sup |e|=\|e\|_{\infty}<\infty
$$

At the same time, from (13)

$$
\dot{V}=s \dot{s}+e \dot{e}=-b_{1} s^{2}-b_{1} e^{2}<\infty
$$

It is obvious that $\dot{s} \in L_{\infty}$ and $\dot{e} \in L_{\infty}$, i.e.

$$
\sup |\dot{s}|=\|\dot{s}\|_{\infty}<\infty, \sup |\dot{e}|=\|\dot{e}\|_{\infty}<\infty
$$

Now let

$$
\begin{gathered}
s_{1}=\theta+\frac{k_{3} m_{2} l^{2}}{k_{1}} \dot{\theta} \\
s_{2}=x+\frac{k_{3} m_{2} l \cos \theta}{k_{2}} \dot{x}
\end{gathered}
$$


Thus

$$
x_{1}=k_{1} s_{1}+k_{2} s_{2}
$$

From our research result (Theorem 1 in [14]), it can be known that $s_{1} \in L_{2}, s_{2} \in L_{2}$ and $s_{1} \in L_{\infty}, \dot{s}_{1} \in L_{\infty}, s_{2} \in L_{\infty}, \dot{s}_{2} \in L_{\infty}$. According to Barbalat's lemma, $\lim _{t \rightarrow \infty} s_{1}=0, \lim _{t \rightarrow \infty} s_{2}=0$, i.e. when $t \rightarrow \infty$,

$$
\begin{aligned}
& \theta+\frac{k_{3} m_{2} l^{2}}{k_{1}} \dot{\theta}=0 \\
& x+\frac{k_{3} m_{2} l \cos \theta}{k_{2}} \dot{x}=0
\end{aligned}
$$

For (15), a positive definite Lyapunov function $V_{2}=\frac{1}{2} \theta^{2}$ is selected and $\dot{V}_{2}=\theta \dot{\theta}=-\frac{k_{3} m_{2} l^{2}}{k_{1}} \dot{\theta}^{2}$ is negative definite. For (16), a positive definite Lyapunov function $V_{3}=\frac{1}{2} x^{2} \quad$ is selected and $\quad \dot{V}_{3}=x \dot{x}=-\frac{k_{3} m_{2} l \cos \theta}{k_{2}} \dot{x}^{2} \quad$ is negative definite when $\theta \in(-\pi / 2, \pi / 2)$. Therefore, the crane system is asymptotically stable to the equilibrium point $(x, \dot{x}, \theta, \dot{\theta})=(0,0,0,0)$.

\section{Simulation Studies}

In this section, the validity of the proposed nonlinear controller by the transport control problem of an overhead crane system. The control objective of the transport control is to transport the load to the required position as fast and as accurately as possible with no free swings. In our simulations, the physical parameters of the overhead crane system in Figure 1 are determined as $m_{1}=1, m_{2}=0.1, l=1$ and gravitational acceleration $g=9.8$. The parameters of the proposed nonlinear controller is selected as $k_{1}=1, k_{2}=15, k_{3}=100, b_{1}=5, b_{2}=5$. The simulation results are shown in Figure 2 and Figure 3. 


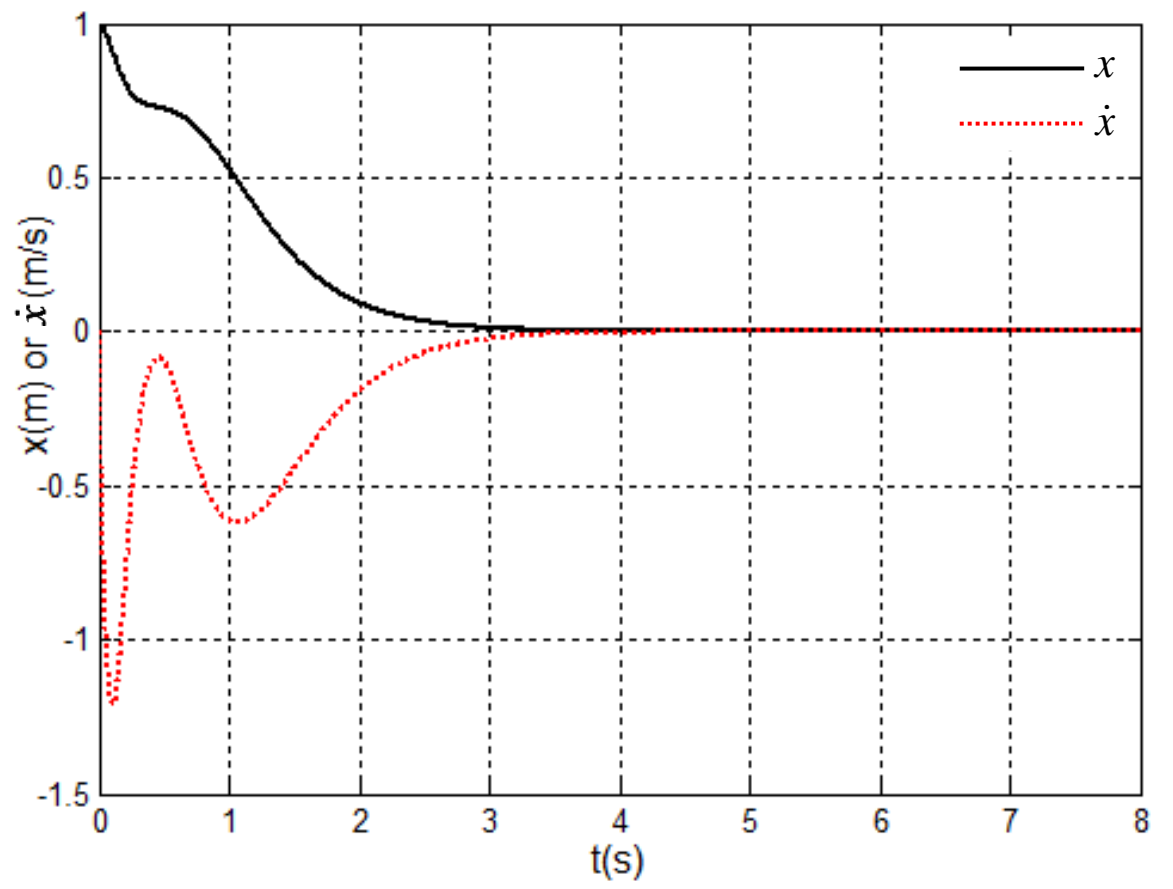

Figure (a). Position and velocity of the cart

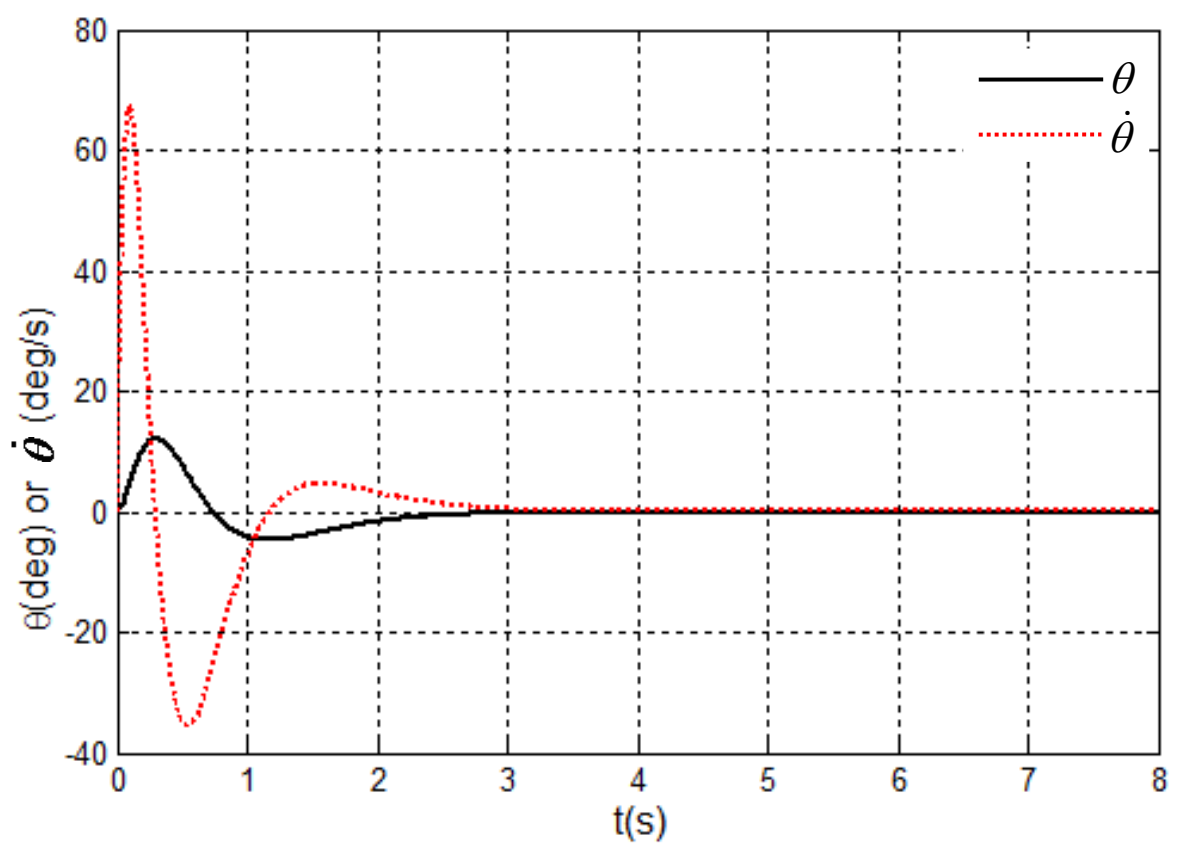

Figure (b). Angle and angle velocity of the pendulum 


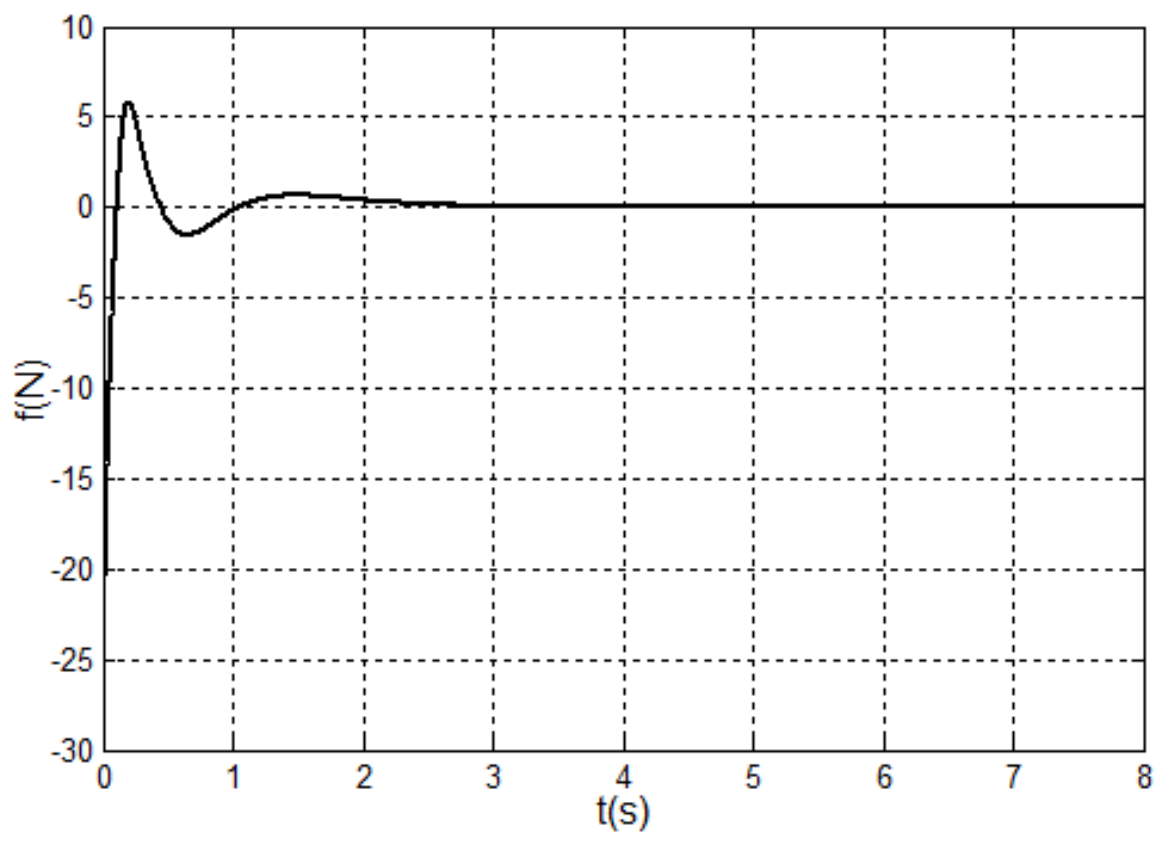

Figure (c). Control force

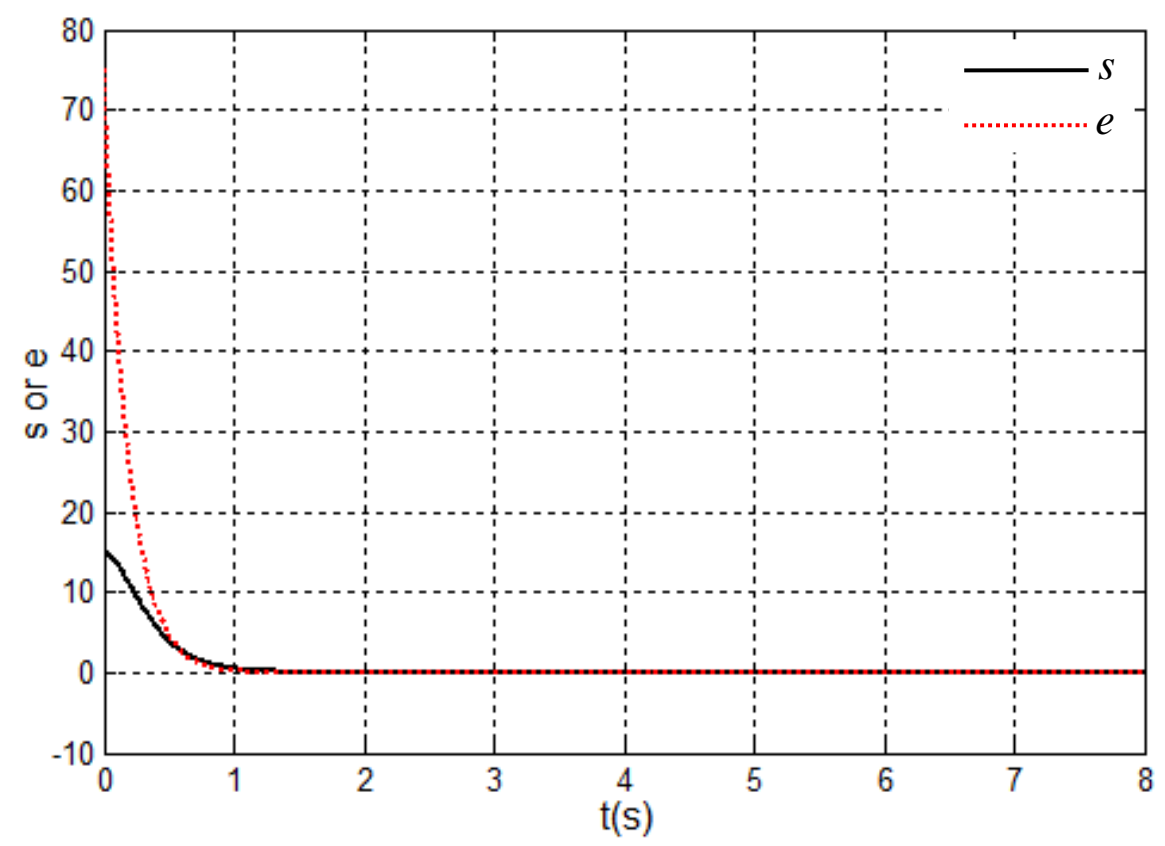

Figure (d). Responses of the nonlinear state variable $s$ and $e$

Figure 2. Simulation results under system initial state $(x, \dot{x}, \theta, \dot{\theta})=(1,0,0,0)$ 


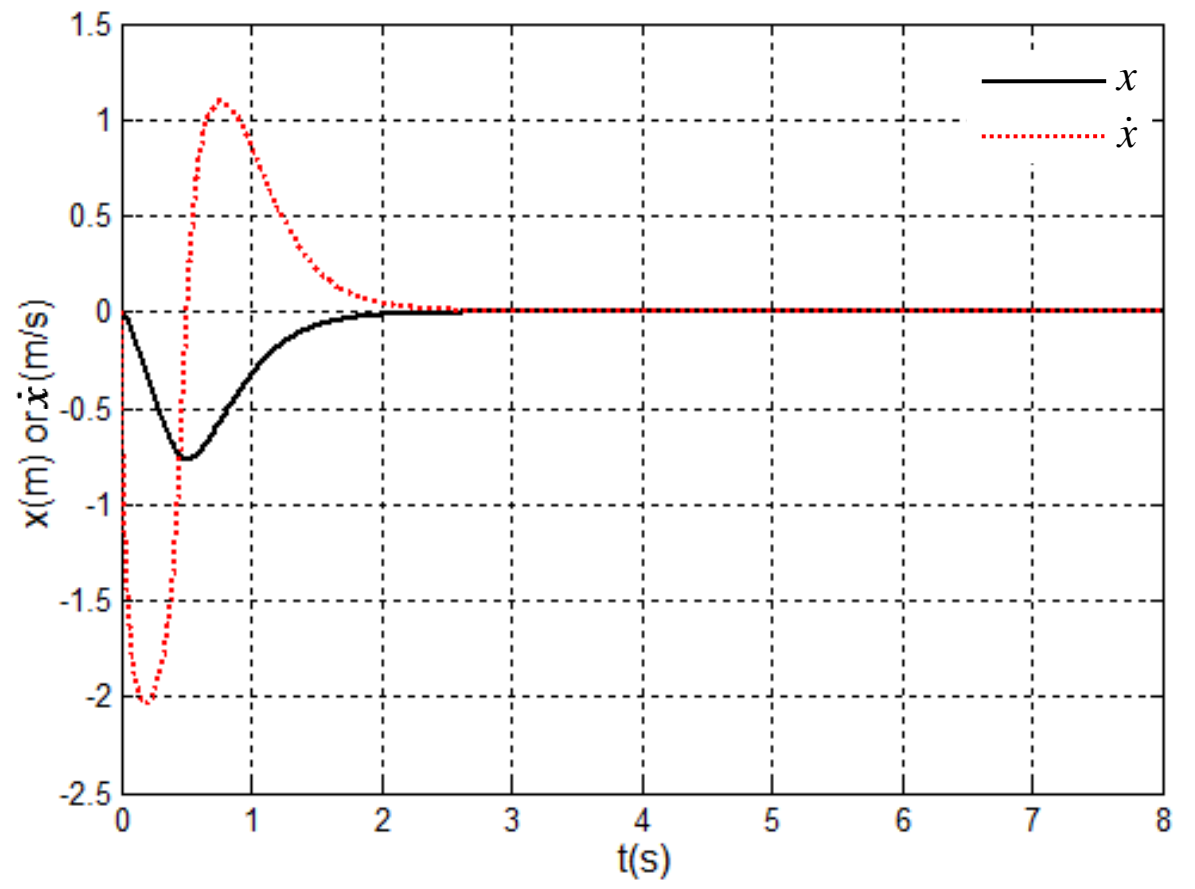

Figure (a). Position and velocity of the cart

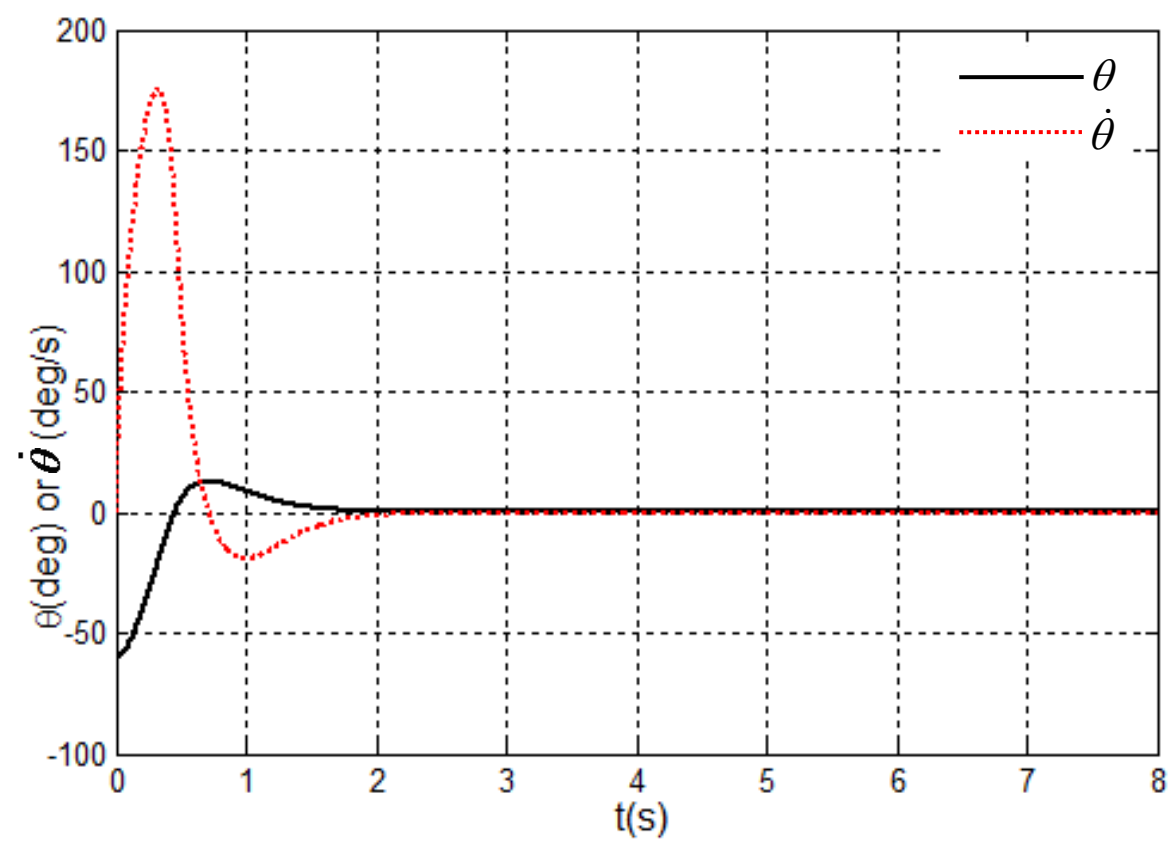

Figure (b). Angle and angle velocity of the pendulum 


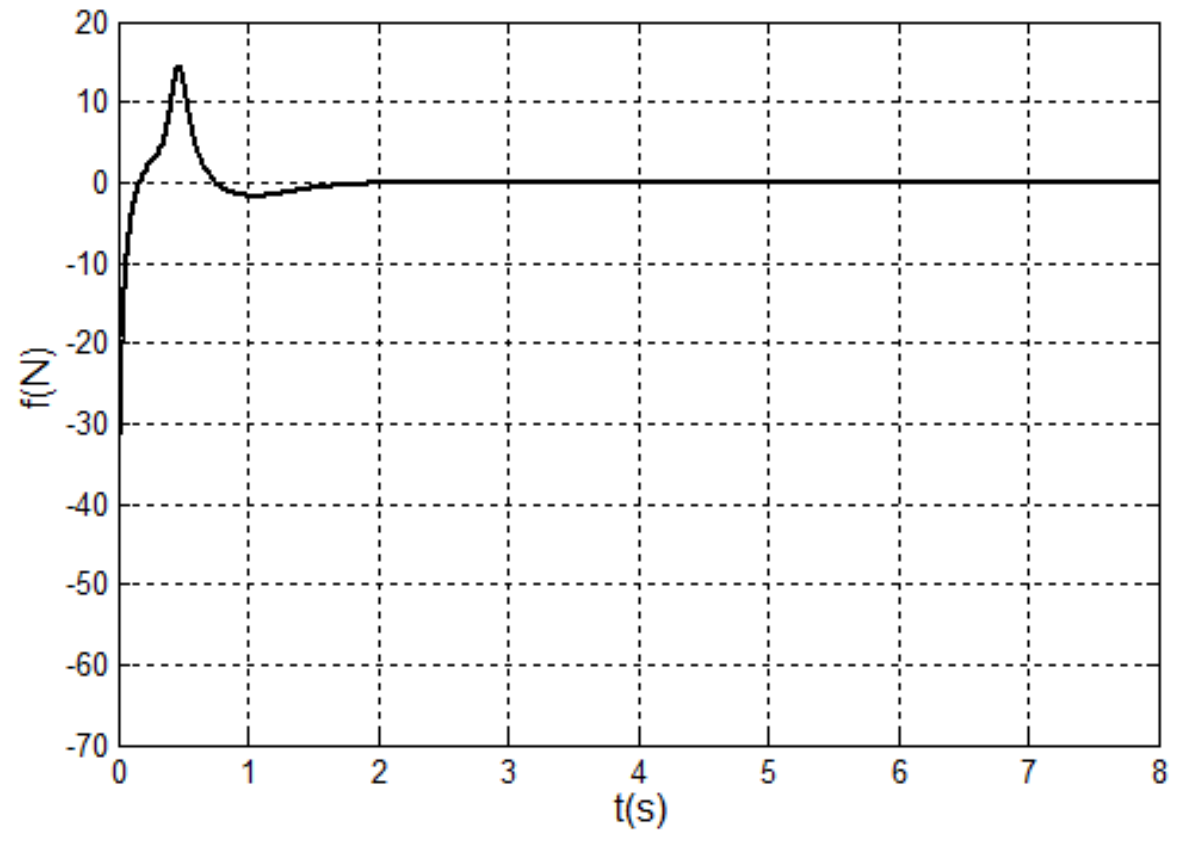

Figure (c). Control force

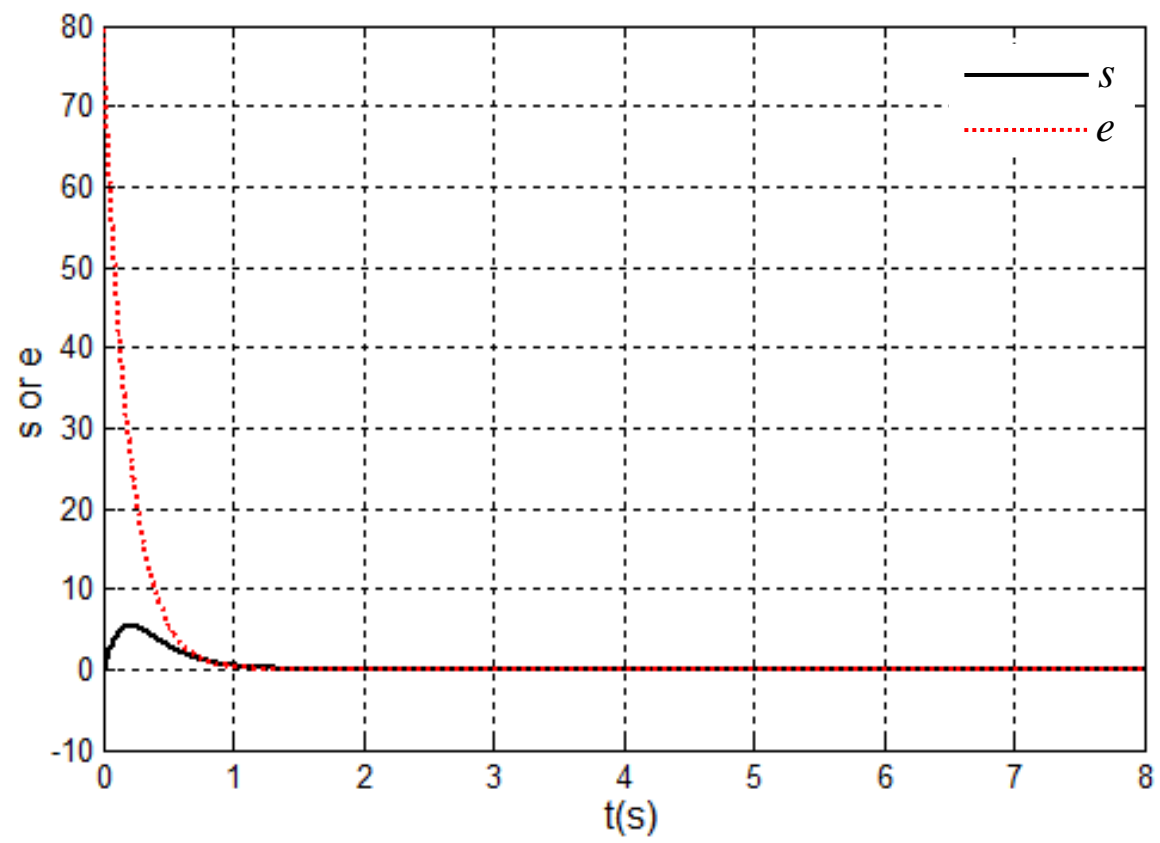

Figure (d). Responses of the nonlinear state variable $s$ and $e$

Figure 3. Simulation results under system initial state $(x, \dot{x}, \theta, \dot{\theta})=(0,0,-\pi / 3,0)$

The simulation results in Figure 2 are obtained under the initial state $(x, \dot{x}, \theta, \dot{\theta})=(1,0,0,0)$ and the simulation results in Figure 3 are obtained under the initial state $(x, \dot{x}, \theta, \dot{\theta})=(0,0,-\pi / 3,0)$. It can be seen form the simulation results and many other 
simulations that the crane system is asymptotically stable under any initial states with the proposed control algorithm. At the same time, it is shown that both the defined nonlinear state variable $s$ in (4) and the $e$ defined in (8) are asymptotically stable. On the other hand, the control performance can be improved through adjusting the parameters of the proposed controller. Lots of simulation experiments show that the parameters $k_{1}, k_{2}$ respectively correspond to the system states $\theta, x$, therefore it is easy to adjust the parameters for an improved system performance.

\section{Conclusions}

Using a collocated partial feedback linearization, the underactuated crane is transformed to a nonlinear system that is used in the control design process. A nonlinear variable is defined and a nonlinear control algorithm is obtained with a selected Lyapunov function. The system stability is studied via a stability theorem and the simulation results show the validity of the proposed control algorithm.

\section{Acknowledgements}

This work is supported by the National Natural Science Foundation of China (61175086) and A Project of Shandong Province Higher Educational Science and Technology Program (No. J13LN25).

\section{References}

[1] J. Q. Yi, N. Yubazaki and K. Hirota, "Anti-swing and positioning control of overhead traveling crane", Information Sciences, vol. 2, no. 155, (2003), pp. 19-42.

[2] K. Yoshida and H. Kawabe, "Design of Saturating Control with a Guaranteed Cost and Its Application to the Crane Control System”, IEEE Transactions on Automatic Control, vol. 37, (1992), pp. 121-127.

[3] Giua, C. Seatzu and G. Usai, "Observer-Controller Design for Cranes via Lyapunov Equivalence", Automatica, vol. 35, (1999), pp. 669-678.

[4] D. T. Liu, J. Q. Yi, D. B. Zhao and W. Wang, "Adaptive Sliding Mode Fuzzy Control for a Two-Dimensional Overhead Crane", Mechatronics, vol. 15, (2005), pp. 505-522.

[5] J. H. Yang and K. S. Yang, "Adaptive Coupling Control for Overhead Crane Systems", Mechatronics, vol. 17, (2007), pp. 143-152.

[6] Y. Fang, W. E. Dixon, D. M. Dawson and E. Zergeroglu, "Nonlinear Coupling Control Laws for an Underactuated Overhead Crane System", IEEE/ASME Transactions on Mechatronics, vol. 8, (2003), pp. 418-423.

[7] N. Sun, Y. Fang, and X. Zhang, "A Novel Nonlinear Coupling Control Approach for Overhead Cranes: Theory and Implementation", Proc. of American Control Conference, (2012), pp.6276-6281.

[8] T. W. Yang and W. J. ÖConnor, "Wave Based Robust Control of a Crane System", Proceedings of IEEE/RSJ International Conference on Intelligent Robots and Systems, vol. 1, (2006), pp. 2724-2729.

[9] W. Wang, X. Liu and J. Yi, "Structure Design of two Types of Sliding-Mode Controllers for a Class of Under-Actuated Mechanical Systems", IET Control Theory \& Applications, vol. 1, (2007), pp. 163-172.

[10] K. W. Choi and J. S. Lee, "Sliding mode control of overhead crane", International Journal of Modelling and Simulation, vol. 31, (2011), pp. 203-209.

[11] Q. H. Ngo and K. S. Hong, "Adaptive sliding mode control of container cranes", IET Control Theory and Applications, vol. 6, (2012), pp. 662-668.

[12] D. Qian and J. Yi, Design of Combining Sliding Mode Controller for Overhead Crane Systems, International Journal of Control and Automation, vol.6, (2013), pp. 131-140.

[13] M. W. Spong. Underactuated Mechanical Systems. Control Problems in Robotics and Automation, Springer Verlag, London, UK, (1997).

[14] W. Wang, J. Yi, D. Zhao and D. Liu, "Design of a stable sliding-mode controller for a class of second-order underactuated systems”, IEE Proc.-Control Theory Appl., vol. 151, (2004), pp. 683-690. 


\section{Authors}

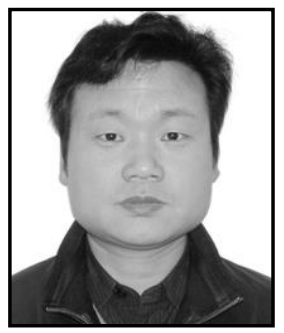

\section{Diantong Liu}

He received the B.S. degree in Electrical engineering from Shandong Institute of Technology, China, in 1994, the M.S. degree in Mechanical Engineering from Tianjin University, China, in 2001, and the Ph.D. degree in Control Engineering from Institute of Automation, Chinese Academy of Sciences, China, in 2004. Currently he is a professor of Institute of Computer Science and Technology, Yantai University. His main research interests include intelligent control, nonlinear system control and mechanical system control.

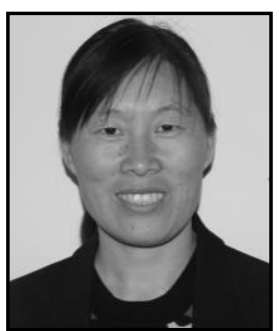

\section{Weiping Guo}

He received the B.S. degree in Electrical engineering from Shandong Institute of Technology, China, in 1996, and the M.S. degree in Control Engineering from Beijing Institute of Technology, China, in 2003. Currently she is a lecturer of Institute of Computer Science and Technology, Yantai University. Her main research interests include chaos and nonlinear system control. 
International Journal of Control and Automation Vol.6, No.6 (2013) 